\title{
Kernos
}

Revue internationale et pluridisciplinaire de religion grecque antique

$13 \mid 2000$

Varia

\section{Yulia USTINOVA, The Supreme Gods of the Bosporan Kingdom. Celestial Aphrodite \& the Most High God}

\author{
Vinciane Pirenne-Delforge
}

\section{OpenEdition \\ Journals}

\section{Édition électronique}

URL : http://journals.openedition.org/kernos/1324

DOI : 10.4000/kernos. 1324

ISSN : 2034-7871

\section{Éditeur}

Centre international d'étude de la religion grecque antique

Édition imprimée

Date de publication : 1 janvier 2000

ISSN : 0776-3824

\section{Référence électronique}

Vinciane Pirenne-Delforge, "Yulia ustinova, The Supreme Gods of the Bosporan Kingdom. Celestial Aphrodite \& the Most High God», Kernos [En ligne], 13 | 2000, mis en ligne le 16 juin 2011, consulté le 24 septembre 2020. URL : http://journals.openedition.org/kernos/1324 ; DOI : https://doi.org/ $10.4000 /$ kernos. 1324 
Dans la ligne de ses travaux antérieurs, l'A. étudie ce qu'il appelle la "religion populaire ", c'est-à-dire ce qui constitue l'activité religieuse du peuple. Il s'en tient dès lors surtout aux traces de cette activité que révèlent les inscriptions. De ce point de vue, ses analyses sont extrêmement fouillées et utiles : elles retouchent notamment le tableau traditionnel d'une période hellénistique plus ou moins «décadente» en regard de la splendeur classique, sous la pression de cultes étrangers et d'associations privées. La religion civique de la période considérée est avant tout caractérisée par la continuité, sciemment orchestrée, avec les périodes antérieures. La prospérité est conçue comme un bienfait lié au respect des traditions, et chaque crise sera suivie d'une volonté de restauration très clairement documentée par les inscriptions. Le recours systématique et presque exclusif à ce type de sources fait la force de cet ouvrage. Mais il en est peut-être aussi la faiblesse : le caractère formel et extrêmement codifié de tels documents ne nous permet pas, sans doute, d'atteindre toutes les dimensions de la Religion in bellenistic Atbens, mais ce qu'ils nous apportent est remarquablement analysé par ce livre.

Vinciane Pirenne-Delforge (Université de Liège)

Yulia Ustinova, The Supreme Gods of the Bosporan Kingdom. Celestial Aphrodite \& the Most High God, Leiden, Brill, 1999. 1 vol. $16 \times 24 \mathrm{~cm}, \mathrm{x}+$ 321 p., 28 pl, 2 cartes (Religions in the Graeco-Roman World, 135). ISBN : 90-04-11231-6.

Pendant les premiers siècles de notre ère, une «Aphrodite Ourania » et un " dieu suprême » ont dominé le panthéon du royaume du Bosphore, c'est-à-dire les régions du littoral nord de la Mer Noire où les Grecs s'étaient installés dès le $\mathrm{vrI}^{\mathrm{e}}$ siècle av. J.-C. Et pendant de longues années, de tels phénomènes religieux ont été essentiellement étudiés par des savants de l'ex-URSS dont les travaux, publiés en russe, restaient largement inconnus des chercheurs « occidentaux». Cette seule remarque montre déjà l'intérêt et l'importance du livre de Yulia Ustinova : non seulement elle offre une synthèse sur des questions qui n'avaient jamais été envisagées dans leur globalité jusqu'ici, mais cette synthèse est abondamment nourrie de la riche documentation archéologique et épigraphique recensée dans une bibliographie forcément mal maîtrisée par ceux qui ne pratiquent pas le russe. De plus, l'analyse de ces données religieuses doit prendre en compte les différentes composantes ethniques de la région (scythe, sindoméotienne, grecque, sarmate et, plus généralement encore, iranienne), ce qui confère à ce livre le statut de "cas d'école " pour l'étude des questions d'influences et de recouvrements religieux, bref tout ce que l'habitude conduit à regrouper sous le terme ambigu de "syncrétisme religieux ».

Après une brève introduction sur l'arrière-plan politique des siècles d'histoire envisagés, la première partie de l'ouvrage porte sur les déesses du Bosphore, en trois chapitres. Ils traitent respectivement des déesses grecques, des déesses locales et enfin de cette déesse toute-puissante que révèle la documentation des premiers siècles de notre ère. En comparaison des maigres attestations des cultes de déesses grecques comme, par exemple, Déméter, Artémis, Hécate ou Athéna, c'est Aphrodite qui a laissé le plus de traces dans les régions étudiées. Du $\mathrm{vi}^{\mathrm{e}}$ au $\mathrm{I}^{\mathrm{er}}$ siècle avant notre ère, le panthéon des colonies grecques du nord de la Mer Noire est dominé par celle que certaines inscriptions appellent Aphrodite Ourania protectrice d'Apatourum et Strabon, Aphrodite Apatouros. La déesse n'étant pas bien attestée dans les métropoles grecques, l'A. en déduit que l'influence locale a dû jouer dans le développement de cette figure, plus que dans le cas des 
autres déesses. Elle comptait au moins quatre sanctuaires importants, à Apatourum, à Phanagorée, à Panticapée et à Cépi, la célébrité des deux premiers dépassant les frontières du royaume. - Les déesses locales du deuxième chapitre sont évoquées au départ du témoignage d'Hérodote sur le panthéon des Scythes, où Aphrodite Ourania est l'interpretatio graeca de la déesse Argimpasa (ou plutôt Artimpasa). L'A. mène une analyse serrée du passage en question (IV, 59) et d'autres, notamment sur les Enareis (IV, 67; I, 105), ces Scythes que l'Aphrodite d'Ascalon aurait effeminés à la suite du pillage de son sanctuaire. L'A. est dès lors amenée à faire l'hypothèse d'influences levantines sur certains rites scythes et, surtout, sur le profil de la déesse Argimpasa, l'Aphrodite Ourania du Bosphore. Les affinités fonctionnelles entre la déesse d'Ascalon, l'Argimpasa des Scythes et Atargatis-Derceto sont bien mises en lumière, mais on a parfois un peu de mal à suivre l'A. dans le détail de tels rapprochements quand elle quitte le terrain de la comparaison fonctionnelle pour celui d'une influence historiquement attestée. Il faut toutefois souligner le cas particulier de la dédicace de la reine Comosarye $\left(\mathrm{rv}^{\mathrm{e}}\right.$ siècle av. J.-C.) " aux puissants dieux Sanerges et Astara ", cette dernière faisant irrésistiblement penser à Astarté : mais qu'en déduire d'un point de vue historique ? Y a-t-il eu « influence », « assimilation », « emprunt » ? L'A. s'en tient à quelques hypothèses. Par ailleurs, les racines iraniennes d'Argimpasa sont envisagées et la comparaison avec Anahita est extrêmement éclairante. L'iconographie des déesses scythes est ensuite longuement analysée : déesse anguipède, déesse aux jambes faites de rameaux enroulés ou de serpents, déesse ailée, déesse aux bras levés, déesse entourée de jeunes gens, autant de figurations qu'il est parfois difficile d'identifier, mais que l'A. ramène avec beaucoup d'arguments intéressants à deux divinités principales : Argimpasa et l'anonyme déesse anguipède seraient ainsi toutes deux Potniai therôn, protectrices des chevaux, incarnations de l'arbre de vie, chthoniennes et célestes en même temps. La déesse anguipède serait plus proche d'une bestialité primitive et des forces élémentaires de la nature, et donc subordonnée à Aphrodite Ourania, davantage en prise sur les affaires humaines, jusqu'à devenir détentrice du pouvoir et dès lors la protectrice du roi. - C'est le troisième chapitre qui aborde cette évolution : alors que, jus qu'au I $^{\text {er }}$ siècle avant notre ère, on pouvait parler d'une prédominance d'Aphrodite par rapport aux autres déesses grecques, à partir de ce moment-là, c'est une véritable domination qui semble se faire jour, et l'A. analyse judicieusement cette situation en rapport avec l'iranisation croissante du Bosphore plus qu'en fonction d'un développement du culte grec de la déesse. Cela signifie qu'Aphrodite Ourania a évolué vers une synthèse entre l'Aphrodite Ourania protectrice d'Apatourum très populaire depuis au moins $\mathrm{le}_{\mathrm{vI}} \mathrm{e}^{\mathrm{e}}$ siècle avant notre ère et la grande déesse locale «scytho-maéto-sarmate » à laquelle elle était identifiée par les Grecs et les indigènes depuis le $\mathrm{v}^{\mathrm{e}}$ siècle. De cet amalgame de cultes aux fonctions similaires est née une divinité toute-puissante, régnant sur ce monde et sur l'autre. Certains traits grecs ont été conservés : le nom, l'iconographie hellénistique et une association privilégiée avec Éros, tandis que les traits locaux se retrouvaient dans le patronage de la royauté, dans la protection des entreprises militaires et des morts.

La deuxième partie de l'ouvrage étudie l'autre grande divinité du Bosphore au début de notre ère, l'anonyme Theos Hypsistos. La première inscription portant son nom date du début du $\mathrm{I}^{\mathrm{er}}$ siècle de notre ère; elle est suivie d'une série d'autres au milieu du II $^{\mathrm{e}}$ siècle pour arriver à l'extension maximale du culte au siècle suivant, du moins en regard de notre documentation. À ce dieu sont liées des associations très hiérarchisées organisées en thiases, surtout actifs à Tanaïs et à Panticapée. Ce «dieu suprême » a longtemps été étudié selon trois axes d'interprétation : on y voyait le dieu du judaïsme - ou un dieu influencé par des 
sympathies juives, - ou le résultat d'influences thrace, ou enfin un dieu d'origine iranienne. L'A. a dès lors entrepris une remarquable réévaluation de ce dossier complexe. Une analyse très détaillée des inscriptions concernant ces associations cultuelles a permis de bien poser les problèmes, en reconnaissant les apories dues à la nature de la documentation. D'autre part, l'hypothèse d'une forte influence du judaïsme dans ces régions a été balayée par une analyse philologique très fine de l'expression $\sigma \varepsilon \beta o ́ \mu \varepsilon v o r ~(\varphi \circ \beta o u ́ \mu \varepsilon v o r)$ tòv $\theta \varepsilon o ́ v$ tant en milieu juif que chrétien et païen : rien ne permet de déduire de telles formules associées aux thiases du Theos Hypsistos un quelconque prosélytisme juif particulièrement actif dans le royaume du Bosphore. Quant à la prétendue influence de Sabazios sur le Theos Hypsistos, l'A. montre que le dossier est vraiment très pauvre et qu'il convient de rester très prudent en cette matière. Enfin, c'est vers l'Iran que se tourne l'analyse de ce dieu anonyme : l'A. rappelle avec force le caractère semibarbare de la cité de Tanaïs, soumise à des influences iraniennes croissantes, comme l'attestent l'iconographie du dieu-cavalier et l'intégration de l'ensemble des habitants mâles de la cité dans les thiases du Theos Hypsistos, ce qui rappelle la tradition iranienne des sociétés guerrières (Männerbünde). Ce " dieu suprême " est donc sans doute une divinité complexe, synthétisant les traits de divers dieux : le dieu solaire honoré par les populations iraniennes des steppes de la Russie du sud, qui peut avoir été associé à Mithra, et aussi le dieu cavalier des Scythes et des Sindo-méotiens.

Les deux volets de cet ouvrage sont précis et abondamment documentés. On suivra l'A. sans aucune difficulté dans la révision très utile de toute une série de dossiers mal connus et insuffisamment approfondis jusque-là. Mes seules réserves tiennent à certaines interprétations trop peu assurées, pour lesquelles le recours à des « étiquettes » toutes faites peut poser problème : par exemple, les notions de chthonien et d'ouranien sont insuffisamment définies, notamment le double rapport de la catégorie du «chthonien » à la fertilité de la terre et au monde des morts, sans induire ipso facto des espérances pour l'au-delà (p. 33-34; 97); le double pilier hermaïque d'Aphrodite retrouvé au Bosphore évoque-t-il vraiment l'androgynie de la déesse ? (p. 37-38); la notion d' « arbre de vie » qui est une sorte de « doublet » de la déesse est trop floue (p. 116); l'identité du personnage mâle, avec ou sans cheval, faisant face à la déesse ou à cet « arbre de vie » est également problématique, et ce problème traverse l'ensemble de l'ouvrage : à la p. 115, c'est un dieu, à la p. 119, c'est un mortel divinisé, identifié à un héros ou à un dieu, et même le roi recevant l'investiture et l'apothéose tout à la fois; p. 266, le cavalier face à la déesse est redevenu un dieu); le thème du bieros gamos supposé entre la déesse et ce personnage est également problématique compte tenu de la seule iconographie disponible (p. 116, 148; aux p. 118, 119, 145sq., il s'agit d'une "communion " qui peut être conçue comme une union matrimoniale); DionysosSabazios (p. 244) est une invention d'auteurs modernes : la documentation ancienne connaît Sabazios et Zeus Sabazios, mais pas ce Dionysos-Sabazios; quant à la corne d'abondance que porterait la figure féminine de la partie supérieure de la splendide plaque de Karagodeouashkh (p. 123 et 159), il semble davantage s'agir d'un pan du vêtement qui revient devant le personnage ( $c f$. M. Daumas, Cabiriaca, pl. 18, 1: ce livre est recensé ici-même). - Ces remarques ne remettent nullement en cause l'orientation très judicieuse des analyses de l'A. Elles montrent simplement la complexité des dossiers, surtout iconographiques, que l'A. a abordés avec un souci d'exhaustivité qui donne une grande force aux convergences qu'elle dégage, et avec la volonté, parfois un peu téméraire, de faire parler au maximum de tels « livres d'images sans textes». 